\title{
Surgical repair of diversion of inferior vena cava into the left atrium nine years after surgical repair of atrial septal defect
}

\author{
Alaa Hijazi ${ }^{1}$, Hülya Çiçekcioğlu ${ }^{2}$, Emir Erol ${ }^{1}$, Ferit Çiçekçioğlu ${ }^{1}$
}

${ }^{1)}$ Department Of Cardiovascular Surgey, Turkey Yuksek Ihtisas Education And Training Hospital, Ankara, Turkey

$\left.{ }^{2}\right)$ Department Of Cardiology, Ankara Numune Education And Training Hospital, Ankara, Turkey

\section{Summary}

Although surgical repair of ASD is fairly safe and a routine procedure very rarely it may be complicated to diversion of inferior vena cava (IVC) into the left atrium. In this report we present a 30 year old male patient who had undergone surgical repair of ASD nine years before he came to our clinic. An echocardiogram was performed and revealed diversion of inferior vena cava into left atrium. Surgical repair of this complication was performed by reconstructing the opening of the IVC and redirecting it into the right atrium.

Keywords: Atrial septal defect, ciyanosis, diversion of inferior vena cava.

\section{Introduction}

Atrial septal defect (ASD) is the third most common congenital cardiac malformation. ${ }^{(1)}$ Although surgical repair of ASD is fairly safe and a routine procedure very rarely it may be complicated to diversion of inferior vena cava (IVC) into left atrium. Diversion of inferior vena cava into left atrium; although unusual; remains a cause of morbidity following repair of ASD.
This leads to dyspnea and hypoxemia which may present immediately postoperative or several years later. In this report we present a 30 year old male patient who started suffering from mild dyspnea and cyanosis on effort one year after surgical repair of ASD. He was brought to the emergency clinic when he underwent a trauma nine years after the closure of ASD, and on performing complete blood count his hematocrit was $\% 65$ and hemoglobin $20 \mathrm{mg} / \mathrm{dl}$. An echocardiogram 
was performed and revealed diversion of inferior vena cava into left atrium. Surgical repair of this complication was performed by reconstructing the opening of the IVC and redirecting it into the right atrium.

\section{Case}

A 30 year old male had undergone surgical repair of ASD in another hospital nine years ago before he presented to our outpatient clinic. One year after the first surgical repair of his ASD he started suffering from perioral cyanosis and dyspnea on effort. The patient neglected these complaints till he fell down from a tree nine years after the first surgery. He was brought to the emergency clinic of another hospital and on performing complete blood count (CBC) his hematocrit was $\% 65$ and hemoglobin $20 \mathrm{mg} / \mathrm{dl}$.

An echocardiogram was performed and revealed diversion of inferior vena cava into left atrium. Then he was referred to our outpatient clinic. His blood pressure was $110 / 70 \mathrm{mmgh}$ and heart rate was $87 / \mathrm{min}$. On physical examination of the patient, mild central cyanosis and clubbing of the fingers of his hands were observed. There was $2 / 6$ systolic ejection murmur with a fixed split S2. Cardiac catheterization was performed as well and confirmed the diagnosis of diversion of inferior vena cava into left atrium. Surgical repair of this complication was performed by reconstruction of the opening of the IVC and redirecting it into the right atrium.
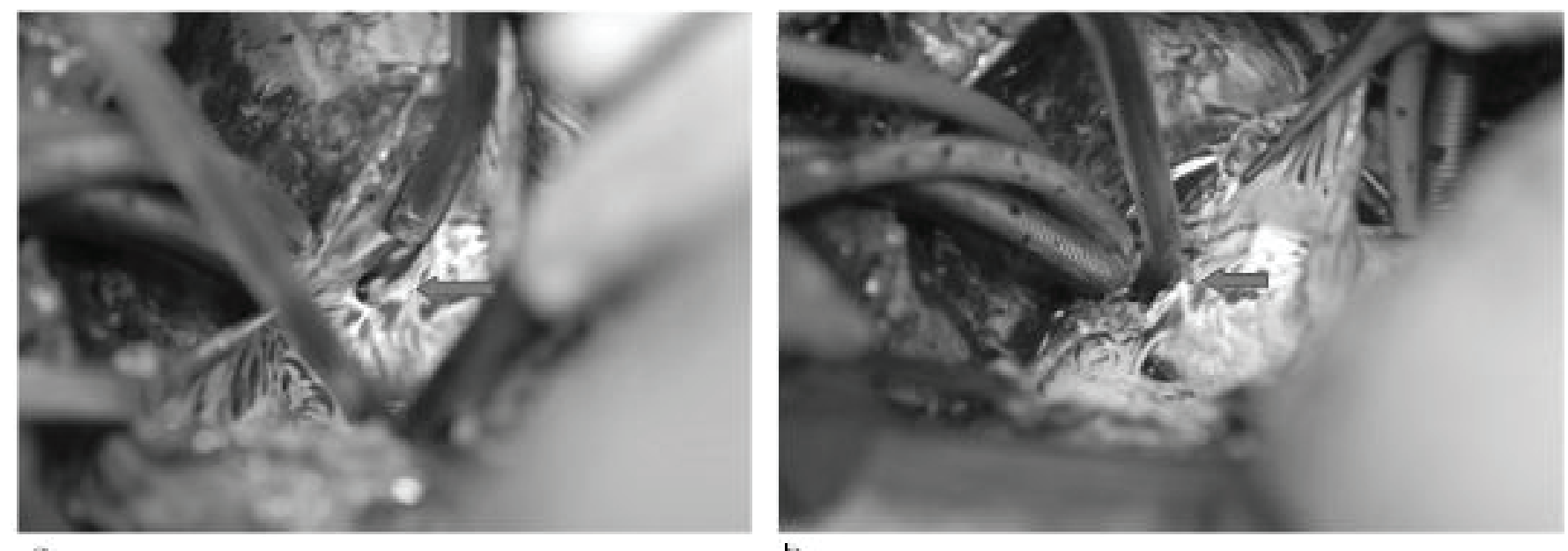

Figure 1. A residual ASD (arrow) and the disappearance of the orifice of the IVC in the right atrium surface. $B$ the upper part of the IVC after being dissected and observing the diversion to the left atrium (arrow).

\section{Surgical technique:}

After re-median sternotomy and dissection the adhesions, aortobicaval cannulation was performed. The purse sutures were place on the IVC and SVC so the tow caval cannules were inserted directly into the IVC and SVC. The Superior and inferior vena cava were snared by the tapes. Cardiopulmonary bypass started and the ascending aorta cross clamped. Ante grade blood cardioplagia was administered and moderate hypothermia established, thus physiological cardiac arrest performed. We stained the inferior and superior vena caval snares. On opening the right atrium we found that the orifice of the IVC had completely occluded with a scar tissue which we thought that it had been formed by the healing process. On making an incision over the scar tissue we observed the diversion of the IVC into the LA and there was a residual ASD which was closed by primary sutures (fig.1a). We dissected the upper part of the IVC and found its opening into the left atrium as seen in figure $1 \mathrm{~b}$. The cannules of the IVC was removed and total circulatory arrest established. The upper part of the IVC was reconstructed by a Dacron graft patch and redirected into the right atrium as seen in Figure 2.

The cannule was re-inserted into the IVC and total circulatory arrest finished. The period of total circulatory arrest was 8 minutes. The right atriotomy was closed and the cross clamp removed. After recovering from the hypothermia, weaning from CPB was uneventful and the sterontomy closed. Postoperative Po2, So2, Hct 
and, $\mathrm{Hb}$ were $87, \% 98 \% 32$ and $10 \mathrm{mg} / \mathrm{dl}$ respectively. The patient was followed up and no complication was reported and the cyanosis disappeared. He was discharged on the fourth day postoperatively. Two months later he came to our outpatient clinic for control. He had no complaints. Echocardiogram revealed no pathology. His Hct and $\mathrm{Hb}$ were $\% 37$ and $11.5 \mathrm{mg} / \mathrm{dl}$ respectively.

\section{Discussion}

Diversion of inferior vena cava into left atrium complicating surgical repair of ASD is a rare condition. As far as we know few cases were published in the recent years, yet it was more frequently seen before the use of

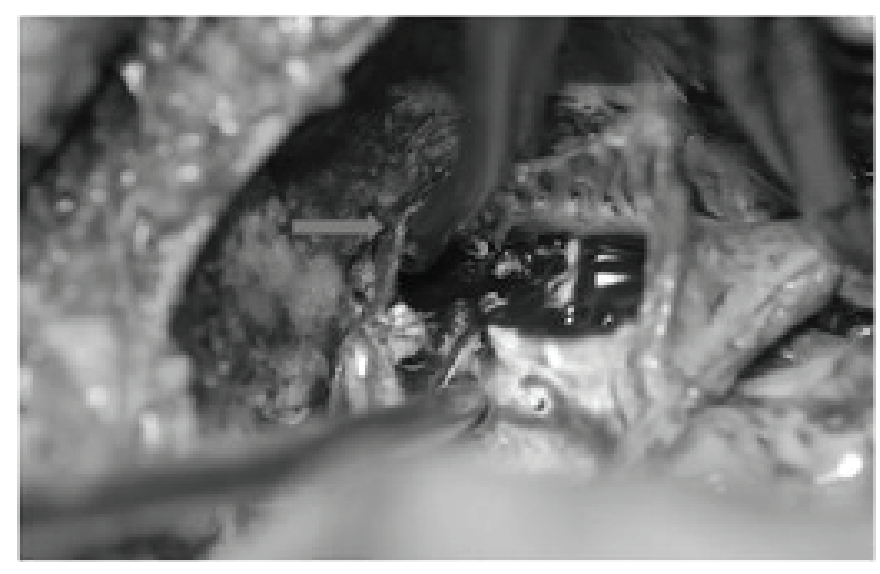

Figure 2. The orifice of the IVC after being reconstructed by a Dacron graft (arrow). cardiopulmonary bypass because time limitations were imposed by only hypothermia and no inflow occlusion. ${ }^{(2)}$

This is an unusual case was discovered nine years after the first operation. Usually the symptoms of this complication appear soon after surgery when the patient becomes cyanotic and hypoxic however some factors such as the relief of pulmonary venous congestion and right ventricular strain by the correction of the left-to-right shunt, the occurrence of only partial diversion of the IVC flow to the LA, and the occurrence of stenosis of the IVC, with collaterals draining to the superior vena cava through an azygos vein may contribute to the appearance of the symptoms of this complication later in life. ${ }^{(3)}$ Reported factors associated with this complication include a large secundum defect or sinus venosus defect, and anomalous pulmonary return into the RA. ${ }^{(4)}$

In our case we observed that the inferior rim of the defect was not included in the first surgical closure. In addition; there was a scar tissue covering the upper part of the IVC on the RA surface so we believe that there was partial diversion of the IVC at first and by healing process complete diversion of the cava occurred gradually. In conclusion, although unusual; diversion of IVC to LA must be considered in cyanotic patients who underwent surgical repair of ASD. 


\section{References}

1. Murphy JG, Gersh BJ, McGoon, Mair, Porter CJ, Ilstrup. Long-term outcome after surgical repair of isolated atrial septal defect. Follow-up at 27 to 32 years. N Engl J Med 1990; 323:1645-50.

2. Staple TW, Ferguson TB, Parker BM. Diversion of the inferior vena cava into the left atrium following atrial septal defect closure. Am J Roentgenol Radium Ther Nucl Med. 1966; 98: 8518.

3. Muath Alanbaei, Luc Jutras, Judith Therrien, and Ariane
Marelli, Iatrogenic cyanosis and clubbing: 25 years of chronic hypoxia after the repair of an atrial septal defect. Can J Cardiol. 2007; 23(11): 901-903.

4. Ellen Thompson, Dennis Moritz, Romaine Perdue, and Silvestre Cansino. Diversion of the Inferior Vena Cava Following Repair of Atrial Septal Defect Causing Hypoxemia. Echocardiography: A Jrnl. of CV Ultrasound \& Allied Tech. Vol. 21, No. 4, 2004.
Received: 22/12/2013

Accepted: $12 / 04 / 2014$

Published: 20/05/2014

Disclosure and conflicts of interest:

Conflicts of interest were not reported.

\section{Corresponding author:}

Dr. Alaa Hijazi

dralaahj@yahoo.com 